\title{
Efficacy of the Jingxin Zhidong Formula for Tic Disorders: A Randomized, Double Blind, Double Dummy, Parallel Controlled Trial
}

\author{
Fei Fan (1D \\ Long $\mathrm{Hao}^{2}$ \\ Si Zhang' \\ Ying Zhang ${ }^{3}$ \\ Zhaoxiang Bian ${ }^{4}$ \\ Xuan Zhang ${ }^{4}$ \\ Qiong Wang ${ }^{5}$ \\ Fei Han (D) ${ }^{\prime}$
}

'Department of Paediatrics, Guang'anmen Hospital, China Academy of Chinese Medical Sciences, Beijing, People's Republic of China; ${ }^{2}$ Department of Paediatrics, Beijing Fangshan District Liangxiang Hospital, Beijing, People's Republic of China; ${ }^{3}$ Centre for EvidenceBased Chinese Medicine, Beijing University of Chinese Medicine, Beijing, People's Republic of China; ${ }^{4}$ Chinese EQUATOR Centre, Hong Kong Chinese Medicine Clinical Study Centre, Chinese Clinical Trial Registry (Hong Kong), School of Chinese Medicine, Hong Kong Baptist University, Kowloon, Hong Kong, People's Republic of China; ${ }^{5}$ Clinical Medical School, Beijing University of Chinese Medicine, Beijing, People's Republic of China
Correspondence: Fei Han

Department of Paediatrics, Guang'anmen Hospital, China Academy of Chinese

Medical Sciences, No. 5, Beixiange Road,

Xicheng District, Beijing, I00053, People's

Republic of China

Tel +86 10-8800 I I 28

Email hf4383@I26.com
Background: The Jingxin Zhidong formula (JXZDF), a traditional Chinese medicine, has been widely used to treat tic disorder (TD) in China. However, its efficacy has not yet been evaluated in a randomized controlled trial. We aimed to compare the effectiveness and safety of JXZDF and aripiprazole in patients with TD.

Methods: In this randomized, double-blind, double-dummy, parallel controlled trial, 120 patients with TD, aged 6-16 years were randomly assigned to receive either JXZDF $(n=60$, $17.6 \mathrm{~g} /$ day) or aripiprazole $(\mathrm{n}=60,10 \mathrm{mg} /$ day) for 12 weeks. The primary outcome was measured using the Yale Global Tic Severity Scale (YGTSS). Adverse events were assessed using the Treatment Emergent Symptom Scale.

Results: JXZDF produced greater improvements than aripiprazole in the following YGTSS subscale scores at the endpoint: total tic scores $(\mathrm{P}=0.004,95 \% \mathrm{CI}$ : $1.085-3.494)$ and total motor scores ( $\mathrm{P}=0.004,95 \% \mathrm{CI}: 0.313-1.739)$. The difference in rate between the groups was no significant $\left(\chi^{2}=0.702\right.$, degrees of freedom $\left.=1, P=0.402\right)$. The overall incidence of adverse events was significantly lower in the JXZDF group than in the aripiprazole group $(0 \%$ vs $6.67 \%, \mathrm{P}<$ $0.001)$.

Conclusion: JXZDF had a better safety profile than aripiprazole, and it was not inferior in terms of clinical efficacy. JXZDF warrants consideration as a potential treatment option for TD.

Trial Registration: CHiCTR, ChiCTR2000039601 (Registered November 2, 2020).

Keywords: tic disorder, Tourette syndrome, Chinese herbal Medicine formula, randomized controlled trial, Jingxin Zhidong formula

\section{Background}

Tic disorder (TD) is a group of childhood-onset neuropsychiatric disorders defined by the presence of one or more motor and/or phonic tics, which are characterised by sudden, rapid, recurrent, non-rhythmic motor movements or vocalisations. ${ }^{1}$ According to the Diagnostic and Statistical Manual of Mental Disorders (DSM)-5, ${ }^{2}$ TD includes Tourette's disorder, persistent (chronic) motor or vocal TD, provisional TD (referred to as transient TD in the DSM-IV), unspecified TD and other specified TD. Current studies have shown that the pathophysiology of TD mainly includes immunological, genetic, neurobiochemical, and inflammation-related processes. ${ }^{1}$ TD frequently co-occurs with various behavioural complications, including attention-deficit hyperactivity disorder (ADHD), obsessive-compulsive behaviour, and obsessive-compulsive disorder $(\mathrm{OCD}){ }^{3}$ these conditions have severe effects on 
the quality of life of patients and their ability to work, resulting in a significant financial burden.

For patients with TD, medical education and psychological support are the first-line treatments. Atypical antipsychotic medications, such as aripiprazole and risperidone, are the preferred pharmacological treatments, because psychological interventions are not effective or available for all patients. ${ }^{4}$ However, these drugs are often associated with adverse effects, such as sedation, weight gain, cognitive dulling, and adverse motor effects. ${ }^{5,6}$ Therefore, alternative therapeutic strategies with equivalent efficacy and superior safety profiles are needed. Traditional Chinese medicines (TCMs), as alternative therapies, have been increasingly used to treat TD in the past decade. Several trials have suggested that TCMs possess therapeutic potential against TD. ${ }^{7,8,10}$

Jingxin Zhidong formula (JXZDF) is an individualised TCM formula pioneered by Prof. Fei Han, a famous Chinese paediatrician. It originates from the therapy theory of tranquilizing mind by nourishing the heart and calming the liver wind. ${ }^{8}$ JXZDF comprises 13 herbal medicines: Zhenzhumu (Concha Margaritifera), calcined Longgu (Os Draconis), Muli (Concha Ostreae), Suan zao ren (Semen Ziziphi Spinosae), Baiziren (Semen Platycladi), Baishao (Radix Paeoniae Alba), Jiangcan (Bombyx Batryticatus), Chaihu (Radix Bupleuri), Niubangzi (Fructus Arctii), Zhiqiao (Fructus Aurantii), Dilong (Lumbricus), Chantui (Periostracum Cicadae), and Baizhi (Angelica Dahurica). These herbs are widely used in the treatment of neuropsychiatric diseases. ${ }^{9-15}$ In TCM practice, drugs containing Bombyx Batryticatus have been used to treat convulsions, ${ }^{12}$ and $O s$ Draconis, Concha Ostreae, and Radix Bupleuri have been used to relieve epileptic symptoms. ${ }^{9}$ All of the above evidence suggests that these herbs might be potentially effective against tics. The choice of herbal medicine is in accordance with the traditional Chinese medicinal theories on the use of herbs.

JXZDF has been Clinically used to treat TD for decades in Guang'anmen Hospital, China Academy of Chinese Medical Sciences (Beijing, China). However, its efficacy has not yet been evaluated in a randomized controlled trial. In this study, we aimed to evaluate the efficacy and safety of pre-made JXZDF granules in children (6-16 years old) with TD.

\section{Methods}

\section{Aim}

The aim of this study was to investigate the efficacy of JXZDF in alleviating TD symptoms and the related impairments.

\section{Trial Design and Setting}

This was a randomized, double blind, double dummy, parallel controlled trial. It was conducted in the Department of Paediatrics, Guang'anmen Hospital, China Academy of Chinese Medical Sciences.

\section{Recruitment}

Patients were recruited between January 2014 and December 2015. All children were outpatients in the Department of Paediatrics of Guang'anmen Hospital and were recruited after study advertisement.

\section{Eligibility Criteria \\ Inclusion Criteria}

The inclusion criteria were as follows: (1) aged 6-16 years; (2) diagnosis of TD (DSM-V); (3) Yale Global Tic Severity Scale (YGTSS) ${ }^{16}$ scores of more than 25 points at the time of screening.

\section{Exclusion Criteria}

The exclusion criteria were as follows: (1) principal diagnosis of ADHD, OCD, Wilson disease, Huntington's disease, rheumatic chorea, epilepsy, intellectual disability, or schizophrenia; (2) tics caused by medications or an undetermined cause; (3) intracranial space-occupying lesions or serious comorbid cardiac, hepatic or renal conditions; (4) a history of allergic reactions to drugs used in this trial; (5) laboratory detection values of hepatic and renal functions that considerably exceeded the normal reference ranges; (6) severe abnormalities detected via electrocardiogram or electroencephalogram tests; and (7) current participation in other clinical trials involving pharmaceutical interventions.

\section{Interventions}

\section{Preparation of Study Medications}

JXZDF and placebo granules were prepared by Jiangyin Tianjiang Pharmaceutical Co. Ltd in strict compliance with the standards of good manufacturing practice. Quality control and assurance were performed by the manufacturer. ${ }^{17}$ A sample specimen has been maintained in the Department of Paediatrics, Guang'anmen Hospital, China Academy of Chinese Medical Sciences. As shown in Table 1, JXZDF contains 13 ingredients, and the quality of these herbs and decoction preparation was in accordance with the Chinese Pharmacopoeia (2005). All ingredients were mixed in appropriate proportions and dissolved in distilled water for $1 \mathrm{~h}$ at room temperature $\left(18^{\circ} \mathrm{C}-26^{\circ} \mathrm{C}\right)$. Decoction (1 h duration) was performed two 
Table I Components of the Jingxin Zhidong Formula

\begin{tabular}{|l|l|l|l|}
\hline Component & Part Used & Amount Used (g) & g/Pack \\
\hline Concha Margaritifera & Shell & 30 & 1.42 \\
Os Draconis & Bone & 30 & 1.42 \\
Concha Ostreae & Shell & 30 & 1.42 \\
Semen Ziziphi Spinosae & Seed & 15 & 0.71 \\
Semen Platycladi & Seed & 15 & 0.71 \\
Radix Paeoniae Alba & Root & 12 & 0.57 \\
Bombyx Batryticatus & Dried larva & 12 & 0.57 \\
Radix Bupleuri & Root & 9 & 0.43 \\
Fructus Arctii & Seed & 9 & 0.43 \\
Fructus Aurantii & Unripe fruit & 6 & 0.28 \\
Lumbricus & Dried body & 6 & 0.28 \\
Periostracum Cicadae & Slough & 6 & 0.28 \\
Angelica Dahurica & Root & 6 & 0.28 \\
\hline
\end{tabular}

times. The decoction obtained was condensed and dried at $60^{\circ} \mathrm{C}$ in a vacuum dryer to produce a granule formation. The granules were packaged in small sachets (each weighing $8.8 \mathrm{~g})$ and stored at room temperature $\left(18^{\circ} \mathrm{C}-26^{\circ} \mathrm{C}\right)$ before use. Samples of JXZDF placebo granules were tested for microbial contamination and deposited with the manufacturer for traceability. The placebo granules were prepared to be identical to JXZDF granules and active aripiprazole in terms of appearance, odour, and colour; they were prepared with dextrin, and food colour and aqueous citric acid were used to mimic the odour and colour of JXZDF. Each aripiprazole tablet (Zhejiang Otzuka Pharmaceutical Co., Ltd., batch number: $\mathrm{H} 20041507)$ contained $5 \mathrm{mg}$ of the active ingredient. All study medications were packaged by the Pharmacy Department of Guang'anmen Hospital.

\section{Study Design}

This was a 12-week, randomized, double-blind clinical trial that involved 12 weeks of therapy and three followup visits every 4 weeks. Patients were randomized to receive either orally administered JXZDF (8.8 g, twice daily) plus aripiprazole placebo tablets $(5 \mathrm{mg}$, twice daily) or aripiprazole (5 $\mathrm{mg}$, twice daily) plus JXZDF placebo granules ( $8.8 \mathrm{~g}$, twice daily). No other medications were administered to the patients during the treatment period. The following assessments were made before patient inclusion: routine blood and urine tests; evaluation of liver and kidney functions; electrocardiogram assessment; documentation of medical history; and physical and neurological examinations. A flow chart of this study is shown in Table 2.

\section{Adherence}

To improve the validity of data, medication compliance was assessed using a variety of methods, including TCM packet counts and weekly telephone follow-up, asking participants whether they were taking the appropriate medication and the reasons for not taking the medication. The participants were asked to return the unused drugs after 12 weeks. We maintained the statistics and records of the participants.

\section{Concomitant Care}

For 12 weeks, the participants were not allowed to use other TCM or western drugs for TD because these drugs could be confused with the effects of JXZDF.

\section{Outcome Measurements}

The primary outcome was measured using the YGTSS and its subscales: total motor (YM) scores (0-25), total phonic (YP) scores (0-25), total tic (YT) scores (sum of YM and YP scores), and tic-related impairment (YI) scores $(0-50)$. The YGTSS has been previously validated in studies conducted among patients with TD. The YGTSS score is the sum of the YT and YI scores. The primary endpoint was the reduction in the YGTSS score and its subscales after 12 weeks of therapy relative to those at baseline. Clinical recovery was indicated by a $\geq 80 \%$ reduction in the YGTSS score, whereas significant improvement was indicated by a reduction of $\geq 50 \%$ and $<80 \%$. The treatment was considered to be effective if the YGTSS score reduction was $\geq 30 \%$ and $<50 \%$, whereas it was considered ineffective if the YGTSS score reduction was $<30 \%$.

Adverse events were assessed at each visit using the Treatment Emergent Symptom Scale, ${ }^{18}$ which included the most commonly encountered adverse effects of aripiprazole and TCM herbs. At the end of the study, physical examinations, blood and urine tests, liver and kidney function evaluations, and electrocardiogram assessments were repeated. All tests were conducted by a single examiner who was blinded to the study outcomes and treatment allocation.

\section{Sample Size Calculation}

In this trial, the effective ratio of JXZDF and aripiprazole was $80 \%$ and $83.3 \%$, with $\alpha=0.05, \beta=0.2$, a target power $=0.8$, and an estimated sample size $=598 .{ }^{19}$ However, owing to limited research funds and the fact that this was 
Table 2 Trial Flow Chart

\begin{tabular}{|c|c|c|c|c|}
\hline \multirow[t]{3}{*}{ TIME POINT } & \multicolumn{4}{|c|}{ Study Period } \\
\hline & \multirow{2}{*}{$\begin{array}{c}\text { Screening/Baseline } \\
-2 \text { to } 0 \text { Days }\end{array}$} & \multicolumn{3}{|c|}{ Treatment Observation } \\
\hline & & 4 Weeks \pm 2 Days & 8 Weeks \pm 2 Days & 12 Weeks \pm 2 Days \\
\hline \multicolumn{5}{|c|}{ Basic medical history collection } \\
\hline $\begin{array}{l}\text { Informed consent } \\
\text { Basic information } \\
\text { Medical history } \\
\text { Concurrent diseases and treatments } \\
\text { Physical examination } \\
\text { Inclusion/Exclusion }\end{array}$ & $\begin{array}{l}\sqrt{ } \\
\sqrt{ } \\
\sqrt{ } \\
\sqrt{ } \\
\sqrt{ } \\
\sqrt{ }\end{array}$ & & & $\sqrt{ }$ \\
\hline \multicolumn{5}{|c|}{ Efficacy observation } \\
\hline YGTSS scores & $\sqrt{ }$ & $\sqrt{ }$ & $\sqrt{ }$ & $\sqrt{ }$ \\
\hline \multicolumn{5}{|c|}{ Safety observation } \\
\hline $\begin{array}{l}\text { Adverse events } \\
\text { Vital Signs } \\
\text { Blood and urine tests } \\
\text { Liver and kidney functions } \\
\text { Electrocardiogram } \\
\text { Electroencephalogram }\end{array}$ & $\begin{array}{l}\sqrt{ } \\
\sqrt{ } \\
\sqrt{ } \\
\sqrt{ } \\
\sqrt{ }\end{array}$ & $\begin{array}{l}\sqrt{ } \\
\sqrt{ }\end{array}$ & $\begin{array}{l}\sqrt{ } \\
\sqrt{ }\end{array}$ & $\begin{array}{l}\sqrt{ } \\
\sqrt{ } \\
\sqrt{ } \\
\sqrt{ } \\
\sqrt{ }\end{array}$ \\
\hline \multicolumn{5}{|c|}{ Others } \\
\hline $\begin{array}{l}\text { Random allocation } \\
\text { Distribution of medications }\end{array}$ & $\begin{array}{l}\sqrt{ } \\
\sqrt{ }\end{array}$ & $\sqrt{ }$ & $\sqrt{ }$ & \\
\hline
\end{tabular}

Note: "ل", the task was performed in that period.

Abbreviation: YGTSS, Yale Global Tic Severity Scale.

a single-centre study, the number of outpatients could not meet the demand of the sample size; only 120 patients were included in the study.

\section{Randomisation and Blinding}

Patients who met the inclusion criteria were assigned to the JXZDF group or the aripiprazole group at a ratio of 1:1 using the PROC PLAN program of SAS package (SAS for Windows, Version 6.12, SAS Institute Inc., Cary, NC). The trial medications were packed in boxes marked with random numbers, and the appearance of all packages was identical. The random codes designating group allocation were sealed in opaque envelopes and kept by the Clinical Efficacy Evaluation Centre of Guang'anmen Hospital, China Academy of Chinese Medical Sciences, which was not associated with the investigator's team. Participants, investigators, and personnel responsible for data entry and analysis were all blinded to group allocation.

\section{Statistical Analyses}

The demographic and clinical characteristics of the patients are presented as mean, standard deviation, and $95 \%$ confidence interval $[\mathrm{CI}]$. Normality of the data was tested using the Shapiro-Wilk test. Comparisons between groups were made using the independent sample $t$-test and repeated measures analysis of variance (ANOVA), provided that the data were normally distributed. Otherwise, Wilcoxon rank-sum test was used as the alternative method. Categorical data were compared using the $\chi^{2}$ test or Fisher's exact test. Simple-effects analyses were performed when we observed an interaction, otherwise we observed main effects. Statistical significance was defined as two-tailed $\mathrm{p}<0.05$. According to the intent-to-treat principles, the last observation was used for patients whose data were not observed throughout the study for statistical estimation and making inferences. ${ }^{20}$ In this study, missing data were calculated using the regression imputation method. All statistical analyses were conducted 
using SPSS software program (SPSS for Windows, Version 19.0. Chicago, SPSS Inc.).

\section{Ethical Considerations}

This randomized controlled trial was retrospectively registered at the Chinese Clinical Trial Registry (ChiCTR2000039601; November 2, 2020). The study protocol was approved by the Ethics Committee of the China Academy of Chinese Medical Sciences Guang'anmen Hospital and conducted in accordance with the World Medical Association Declaration of Helsinki. $^{21,22}$ The primary guardians of all enrolled children provided written informed consent; written consent was also obtained from children over 8 years of age. The sample size and all outcome measures were determined before study initiation and were not modified thereafter.

\section{Results}

\section{Participant Characteristics}

A total of 632 patients with TD were screened between January 2014 and December 2015; 175 patients met the inclusion criteria and had YGTSS scores of 25-70 at the time of screening. Among these patients, 120 agreed to participate in the trial. There were 60 patients in each group. Their average age was $9.23 \pm 2.60$ years, and 108 of the patients were males $(90 \%)$. The median duration of illness was 24 (interquartile range, 12-36) months. There were no significant differences in age, sex ratio, or duration of illness between the treatment groups. A total of 109 (90.8\%) patients completed the 12-week treatment period, whereas 11 patients discontinued treatment due to intolerable adverse effects $(n=2)$; loss to follow-up ( $n=7)$, that is, inability to return for follow-up and emigration; and personal reasons, which the patients refused to disclose $(n=2)$ (Figure 1). The baseline characteristics and assessment scores of the patients are summarised in Table 3. There were no significant differences in baseline variables between the groups.

\section{Comparison of Treatment Outcomes Between the Groups}

The treatment outcomes are summarised in Table 4. There were no significant differences in the assessment scores between the groups at baseline. Throughout the intervention period, the YT (Figure 2), YM (Figure 3), YP (Figure 4), and YI (Figure 5) scores decreased in both groups at each follow-up assessment. The YT (Figure 2) and YM (Figure 3) scores were lower in the JXDZF group than in the aripiprazole group at week 12. Both YT and

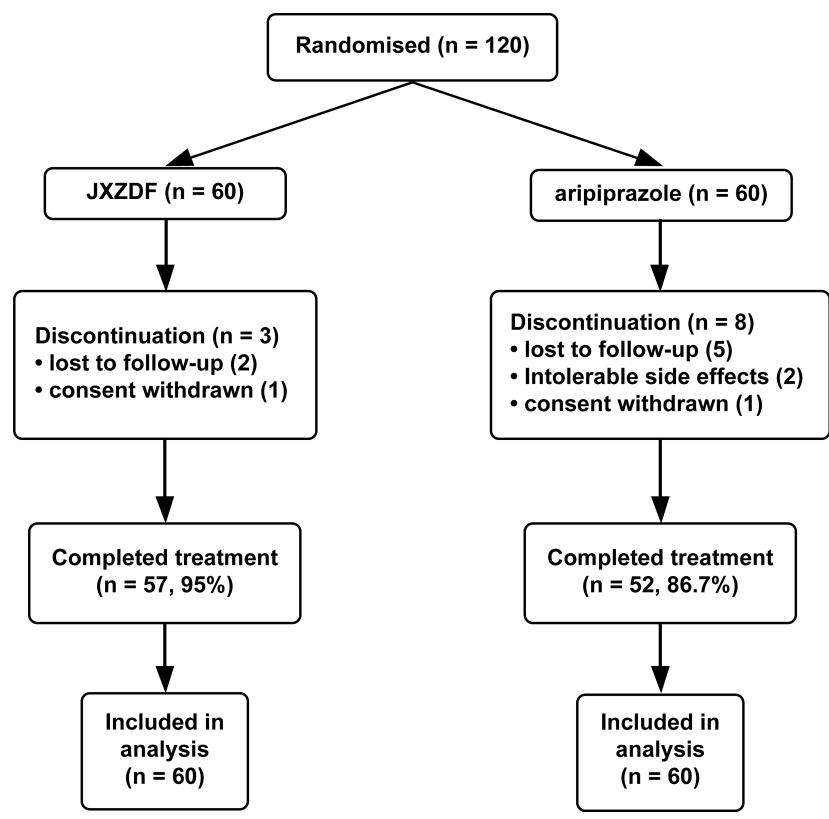

Figure I Flowchart of patient screening and recruitment. Abbreviation: JXZDF, Jingxin Zhidong formula.

YM scores were significant differences between the groups at week 12 (Supplementary Tables 1-6) However, no significant differences were observed between the groups at week 4 or week 8 .

Clinical recovery or significant improvement was observed in 93.3\% (56/60) of JXZDF-treated patients; this did not differ significantly from the aripiprazoletreated patients $\left(96.7 \%, 58 / 60 ; \chi^{2}=0.702\right.$, degrees of freedom $=1, \mathrm{p}=0.402$ ).

Table 3 Baseline Characteristics of Patients with TD

\begin{tabular}{|l|c|c|c|}
\hline Variable & \multicolumn{1}{|c|}{$\begin{array}{c}\text { JXZDF } \\
(\mathbf{n}=60)\end{array}$} & $\begin{array}{c}\text { Aripiprazole } \\
(\mathbf{n}=60)\end{array}$ & P \\
\hline Characteristic & $9.67 \pm 2.86$ & $8.80 \pm 2.25$ & 0.068 \\
\hline Age, y & $6 / 54(10 / 90 \%)$ & $6 / 54(10 / 90 \%)$ & 1.000 \\
\hline Gender (female/male) & $25.70 \pm 21.76$ & $26.70 \pm 18.40$ & 0.786 \\
\hline Duration of TD, m & $26.25 \pm 7.19$ & $25.15 \pm 7.43$ & 0.438 \\
\hline Baseline assessment scores & $15.75 \pm 3.99$ & $14.82 \pm 3.21$ & 0.161 \\
\hline YT & $10.68 \pm 6.00$ & $10.03 \pm 6.18$ & 0.560 \\
\hline YM & $27.42 \pm 6.07$ & $29.17 \pm 5.61$ & 0.104 \\
\hline YP & & & \\
\hline YI &
\end{tabular}

Abbreviations: JXZDF, Jingxin Zhidong formula; TD, tic disorder; YT, total tic score; YM, total motor score; YP, total phonic score; YI, tic-related impairment scale score. 

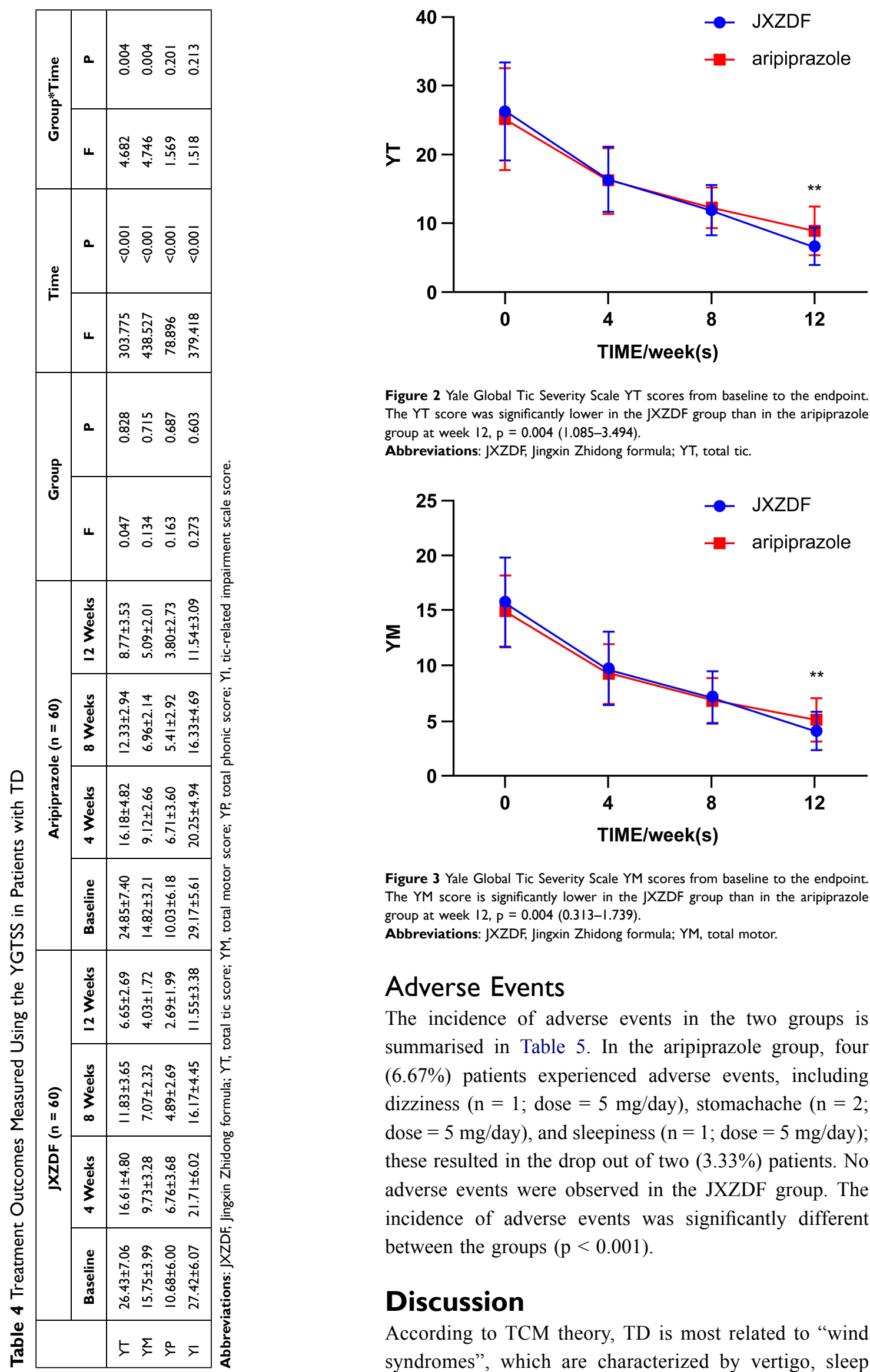

Figure 2 Yale Global Tic Severity Scale YT scores from baseline to the endpoint. The YT score was significantly lower in the JXZDF group than in the aripiprazole group at week 12, $\mathrm{p}=0.004$ (1.085-3.494).

Abbreviations: JXZDF, Jingxin Zhidong formula; YT, total tic.

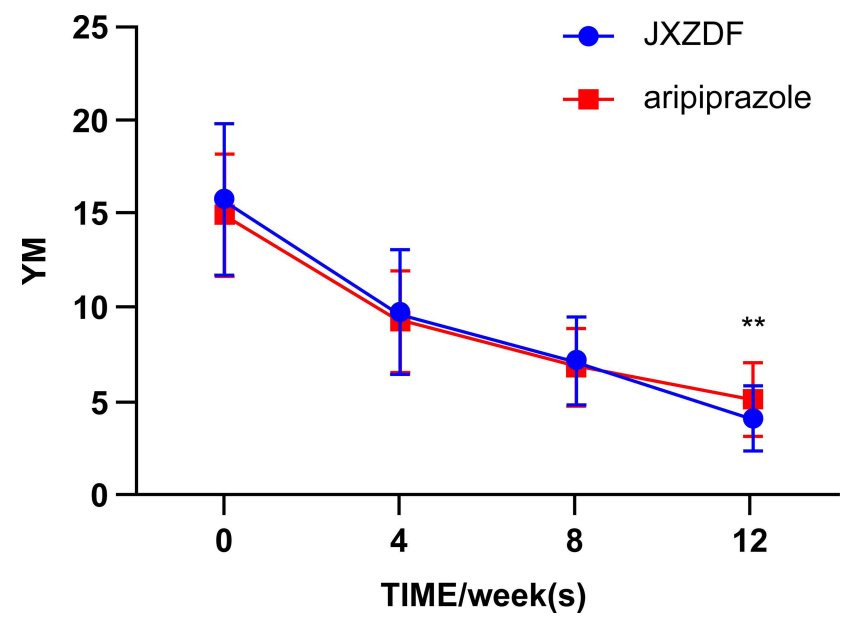

Figure 3 Yale Global Tic Severity Scale YM scores from baseline to the endpoint. The YM score is significantly lower in the JXZDF group than in the aripiprazole group at week 12, $\mathrm{p}=0.004(0.313-1.739)$.

Abbreviations: JXZDF, Jingxin Zhidong formula; YM, total motor.

\section{Adverse Events}

The incidence of adverse events in the two groups is summarised in Table 5. In the aripiprazole group, four (6.67\%) patients experienced adverse events, including dizziness $(\mathrm{n}=1$; dose $=5 \mathrm{mg} /$ day $)$, stomachache $(\mathrm{n}=2$; dose $=5 \mathrm{mg} /$ day $)$, and sleepiness $(\mathrm{n}=1$; dose $=5 \mathrm{mg} /$ day); these resulted in the drop out of two $(3.33 \%)$ patients. No adverse events were observed in the JXZDF group. The incidence of adverse events was significantly different between the groups $(\mathrm{p}<0.001)$.

\section{Discussion}

According to TCM theory, TD is most related to "wind syndromes", which are characterized by vertigo, sleep 


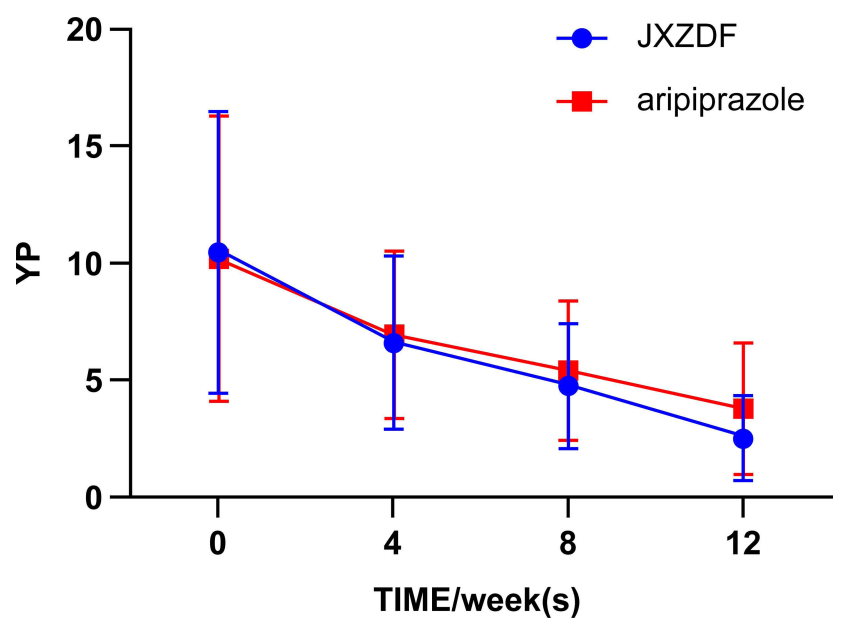

Figure 4 Yale Global Tic Severity Scale YP scores from baseline to the endpoint. Abbreviations: JXZDF, Jingxin Zhidong formula; YP, total phonic.

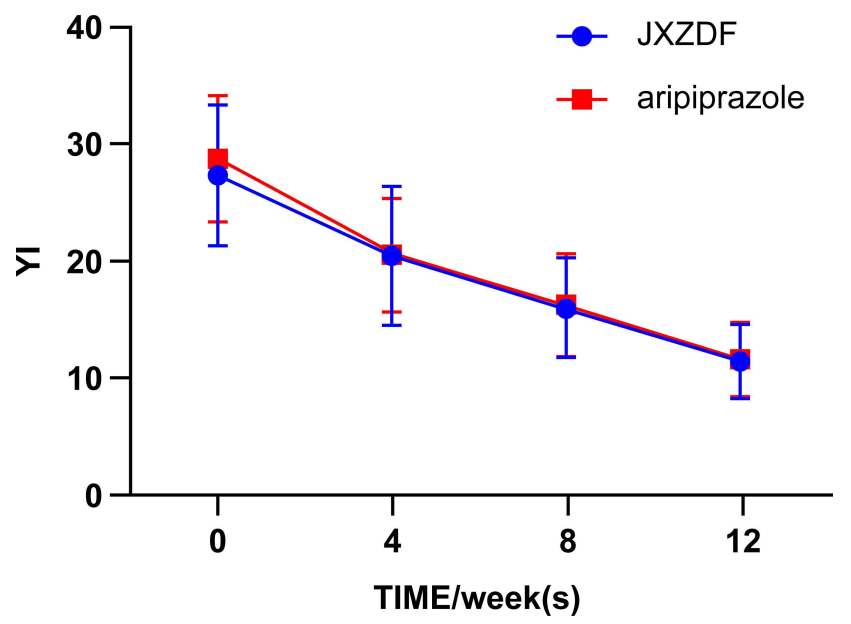

Figure 5 Yale Global Tic Severity Scale YI scores from baseline to the endpoint. Abbreviations: JXZDF, Jingxin Zhidong formula; YI, impairment.

disorders, musculoskeletal paralysis, pruritus, tremor, numbness, and spasm; mainly caused by the lack of spirit preservation and liver wind agitation. ${ }^{23}$ Tranquilizing the mind by nourishing the heart and

Table 5 Incidence of Major Adverse Events in the Two Groups of Patients with Tic Disorders

\begin{tabular}{|l|c|c|c|}
\hline Adverse Events & $\begin{array}{c}\text { JXDZF } \\
(\mathbf{n}=\mathbf{6 0})\end{array}$ & $\begin{array}{c}\text { Aripiprazole } \\
\mathbf{( n = 6 0 )}\end{array}$ & \multirow{2}{*}{} \\
\cline { 2 - 3 } & $\mathbf{n}$ (Case), \% & $\mathbf{n}$ (Case), \% & \\
\hline Adverse events (total) & 0 & $4,6.67$ & $<0.00 \mathrm{I}$ \\
Dizziness & 0 & $\mathrm{I}, \mathrm{I} .67$ & \\
Stomachache & 0 & $2,3.33$ & \\
Sleepiness & 0 & $\mathrm{I}, \mathrm{I} .67$ & \\
\hline
\end{tabular}

Abbreviation: JXZDF, Jingxin Zhidong formula. calming the liver can alleviate the "wind syndromes" according to Huangdi Neijing (Yellow Emperor's Canon) by the Yellow Emperor of China (26952589 BC). Considering the physiological and pathological characteristics of children, mind-soothing and liver wind-extinguishing medicines were selected to alleviate tic symptoms in this study. The results of this study showed that JZXDF was effective in the treatment of TD. The prevalence of TD across China is $6.1 \%{ }^{24}$ Behavioural therapy $^{25}$ and deep brain stimulation ${ }^{26}$ can also be used to treat TD; however, they are not suitable for all patients owing to their high cost and limited applicability to specific disease conditions. In recent years, several studies have demonstrated the efficacy of traditional Chinese herbal medicines in the treatment of psychiatric disorders ${ }^{27,28}$ and explored their potential mechanisms. ${ }^{9-15}$ JXZDF is an individualised TCM formula and has been clinically used to treat TD for decades in China, and it is considered safe and effective.

Although both groups of patients in the present study showed improvements in motor and vocal tics during the treatment, patients in the JXZDF group exhibited significantly lower YT scores and YM subscale scores than those in the aripiprazole group. The proportion of patients in the JXZDF group who achieved a clinical response at week 12 was not statistically different from that in the aripiprazole group. JXZDF was safer than aripiprazole, and fewer patients in the JXZDF group withdrew from this trial.

TD has a multifactorial aetiology and multi-system pathogenesis. The dysfunction of neurotransmitters (eg, dopamine, glutamate, $\gamma$-aminobutyric acid, 5-hydroxytryptamine, and noradrenaline) in the cortico-striato-thalamocortical circuits contributes to TD development. ${ }^{1}$ Among the ingredients in the JXZDF formulation, Radix Bupleuri, Os Draconis, and Concha Ostreae are known to relieve disorders by clearing away heat, tranquilizing the mind, and eliminating excitement. ${ }^{9}$ Several studies have suggested that components derived from these three herbal materials are potentially effective for treating psychiatric disorders, including epilepsy and insomnia. ${ }^{29}$ The active components of Radix Bupleuri and Radix Paeoniae Alba can increase plasma adrenocorticotropic hormone levels and decrease 5-hydroxytryptamine and dopamine levels. ${ }^{30}$ Semen Ziziphi Spinosae modulates the levels of monoamines and amino acid neurotransmitters in the brain. $^{31}$ Furthermore, extracts from Bombyx Batryticatus and Lumbricus possess broad psychotherapeutic properties, as they modulate brain $\gamma$-aminobutyric acid, 
glutamate, and related glutamate receptor activities. ${ }^{14,32}$ Therefore, the efficacy of JXZDF observed in this study might be related to a variety of pharmacological and therapeutic properties of these components. Furthermore, the associations between gut microbiota and neuropsychiatric diseases has received increasing research attention, known as the gut-brain axis. Both clinial and animal studies demonstrated that microbiota have the potential to improve tic syndromes. ${ }^{33-35}$ Notably, several components of the JXZDF, including Semen Ziziphi Spinosae, Radix Bupleuri, Fructus Aurantii and Angelica Dahurica are potentially effective at modulating gut microbiota abundances. ${ }^{36-39}$ Further studies could be designed to assess the gut microbiota to reveal the underlying pharmacological and therapeutic effects of JXZDF for the treatment of TD.

\section{Limitations}

The present study had some limitations. First, due to limited funding, the number of enrolled patients was inadequate; this might have reduced the precision of the results. Nevertheless, significant differences in the outcomes were observed between the JXZDF and aripiprazole groups. Second, differences in the population, including height, weight, genetics, co-occurring behavioural complications, and dietary factors, were not analysed in this study. Moreover, the randomisation method did not consider that the participants in the JXDZF group were slightly older; this is a possible confounding factor. Third, the dosage of the two medications was not adjusted according to the specific clinical conditions of each patient. In general, the initial dose of aripiprazole for children is $2 \mathrm{mg} /$ day, which can be titrated up to $10 \mathrm{mg} /$ day, depending on disease severity. ${ }^{40}$ In this study, we used the maximum dose of aripiprazole recommended for children. This may have resulted in a more rapid efficacy, earlier resistance, and relatively significant adverse effects in the aripiprazole group. In contrast, resistance was not observed in the JXZDF group. This may account for the difference in efficacy between the groups at week 12 . Fourth, this trial involved only 12 weeks of therapy, with a lack of information on the effectiveness and safety of longer JXZDF treatments. Fifth, this study was a singlecentre trial, and only children (aged 6-16 years) were included in this study owing to the study setting of the Department of Paediatrics; further studies are needed to determine the effects of JXZDF on adults. Sixth, this trial lacked an inactive placebo, and the effects observed in both groups could be solely due to the placebo effect. Finally, syndrome differentiation of TCMs was not considered in the present study. Therefore, additional studies are needed to determine whether JXZDF is more beneficial for specific TCM syndrome types.

\section{Conclusions}

The results of this study suggest that JXZDF is noninferior to aripiprazole in terms of its effectiveness to alleviate tic symptoms and the related impairments. However, we demonstrated that JXZDF has a better safety profile. Therefore, JXZDF warrants consideration as a potential therapy for TD.

\section{Acknowledgments}

This study was supported by the institute-level project of the China Academy of Chinese Medical Sciences.

\section{Disclosure}

The authors report no conflicts of interest in this work.

\section{References}

1. Robertson MM, Eapen V, Singer HS, et al. Gilles de la Tourette syndrome. Nat Rev Dis Primers. 2017;3(1):16097. doi:10.1038/ nrdp.2016.97

2. American Psychiatric Association. Diagnostic and Statistical Manual of Mental Disorders (DSM-5). 5th ed. Washington, DC: American Psychiatric Association; 2013.

3. Conte G, Valente F, Fioriello F, Cardona F. Rage attacks in Tourette syndrome and chronic tic disorder: a systematic review. Neurosci Biobehav Rev. 2020;119:21-36. doi:10.1016/j.neubiorev.2020.09.019

4. Roessner V, Eichele H, Stern JS, et al. European clinical guidelines for Tourette syndrome and other tic disorders-version 2.0. Part III: pharmacological treatment [published online ahead of print, 2021 Nov 10]. Eur Child Adolesc Psychiatry. 2021. doi:10.1007/s00787021-01899-z

5. Scahill L, Erenberg G, Berlin CM Jr, et al. Contemporary assessment and pharmacotherapy of Tourette syndrome. Neurorx. 2006;3(2):19 2-206. doi:10.1016/j.nurx.2006.01.009

6. Yang C, Hao Z, Zhang LL, Zhu CR, Zhu P, Guo Q. Comparative efficacy and safety of antipsychotic drugs for tic disorders: a systematic review and Bayesian network meta-analysis. Pharmacopsychiatry. 2019;52(1):7-15. doi:10.1055/s-0043-124872

7. Kim YH, Son CG, Ku BC, Lee HW, Lim HS, Lee MS. Herbal medicines for treating tic disorders: a systematic review of randomised controlled trials. Chin Med. 2014;9(1):6. doi:10.1186/17498546-9-6

8. Bo W, Fei H. Effect and of Jingxin Zhidong Fang on neuropeptides and autoimmunity of twitch disorder children. J Hainan Med Univ. 2013;19(2):247-252. In Chinese.

9. Yang P, Qin Y, Zhu Y, et al. Chaihu-Longgu-Muli decoction relieves epileptic symptoms by improving autophagy in hippocampal neurons. J Ethnopharmacol. 2020;259:112990. doi:10.1016/j.jep.2020.112990

10. Zheng Y, Zhang ZJ, Han XM, et al. A proprietary herbal medicine (5-Ling Granule) for Tourette syndrome: a randomized controlled trial. J Child Psychol Psychiatry. 2016;57(1):74-83. doi:10.1111/ jcpp. 12432 
11. Chen IC, Lin TH, Hsieh YH, et al. Formulated Chinese medicine Shaoyao Gancao tang reduces tau aggregation and exerts neuroprotection through anti-oxidation and anti-inflammation. Oxid Med Cell Longev. 2018;2018:9595741. doi:10.1155/2018/9595741

12. $\mathrm{Hu}$ M, Yu Z, Wang J, et al. Traditional uses, origins, chemistry and pharmacology of Bombyx batryticatus: a review. Molecules. 2017;22 (10):1779. doi:10.3390/molecules22101779

13. Zhao S, Liu Z, Wang M, et al. Anti-inflammatory effects of Zhishi and Zhiqiao revealed by network pharmacology integrated with molecular mechanism and metabolomics studies. Phytomedicine. 2018;50:61-72. doi:10.1016/j.phymed.2018.09.184

14. Cooper EL, Balamurugan M, Huang CY, et al. Earthworms dilong: ancient, inexpensive, noncontroversial models may help clarify approaches to integrated medicine emphasizing neuroimmune systems. Evid Based Complement Alternat Med. 2012;2012:164152. doi:10.1155/2012/164152

15. Wang C, Sun J, Li H, Yang $X$, Liu $H$, Chen J. In vivo anti-inflammatory activities of the essential oil from Radix Angelicae dahuricae. J Nat Med. 2016;70(3):563-570. doi:10.1007/ s11418-016-0978-0

16. Leckman JF, Riddle MA, Hardin MT, et al. The yale global tic severity scale: initial testing of a clinician-rated scale of tic severity. J Am Acad Child Adolesc Psychiatry. 1989;28(4):566-573. doi:10.1097/00004583-198907000-00015

17. Liang D, Yin YH, Miao LY, et al. Integrating chemical similarity and bioequivalence: a pilot study on quality consistency evaluation of dispensing granule and traditional decoction of Scutellariae Radix by a totality-of-the-evidence approach. $J$ Pharm Biomed Anal. 2019;169:1-10. doi:10.1016/j.jpba.2019.02.030

18. Al-Dhaher Z, Kapoor S, Saito E, et al. Activating and tranquilizing effects of first-time treatment with aripiprazole, olanzapine, quetiapine, and risperidone in youth. J Child Adolesc Psychopharmacol. 2016;26(5):458-470. doi:10.1089/cap.2015.0141

19. Tao D, Zhong T, Ma S, Li J, Li X. Randomized controlled clinical trial comparing the efficacy and tolerability of aripiprazole and sodium valproate in the treatment of Tourette syndrome. Ann Gen Psychiatry. 2019;18(1):24. doi:10.1186/s12991-019-0245-3

20. Solomon N, Lokhnygina Y, Halabi S. Comparison of regression imputation methods of baseline covariates that predict survival outcomes. J Clin Transl Sci. 2020;5(1):e40. doi:10.1017/cts.20 20.533

21. World Medical Association. World Medical Association Declaration of Helsinki: ethical principles for medical research involving human subjects. JAMA. 2013;310(20):2191-2194. doi:10.1001/jama.2013.28 1053

22. Song TJ, Leng HF, Zhong LL, Wu TX, Bian ZX. CONSORT in China: past development and future direction. Trials. 2015;16(1):243. doi:10.1186/s13063-015-0769-z

23. Kyungjin L, Bumjung K, Youngmin B, et al. Review on the characteristics of liver-pacifying medicinal in relation to the treatment of stroke: from scientific evidence to traditional medical theory. $J$ Tradit Chin Med. 2018;38(1):139-150. doi:10.1016/j.jtcm.2018.01.003

24. Yang C, Zhang L, Zhu P, Zhu C, Guo Q. The prevalence of tic disorders for children in China: a systematic review and meta-analysis. Medicine. 2016;95(30):e4354. doi:10.1097/MD.00 00000000004354

25. McGuire JF, Ginder N, Ramsey K, et al. Optimizing behavior therapy for youth with Tourette's disorder. Neuropsychopharmacology. 2020;45(12):2114-2119. doi:10.1038/s41386-020-0762-4
26. Baldermann JC, Schüller T, Huys D, et al. Deep brain stimulation for Tourette-syndrome: a systematic review and meta-analysis. Brain Stimul. 2016;9(2):296-304. doi:10.1016/j.brs.2015.11.005

27. Deng H, Adams CE. Traditional Chinese medicine for schizophrenia: a survey of randomized trials. Asia Pac Psychiatry. 2017;9(1):1. doi:10.1111/appy.12265

28. Yang WT, Zheng XW, Chen S, et al. Chinese herbal medicine for Alzheimer's disease: clinical evidence and possible mechanism of neurogenesis. Biochem Pharmacol. 2017;141:143-155. doi:10.1016/ j.bcp.2017.07.002

29. Wang X, Ju J, Li J, Fan Y, Xu H. Chaihu Longgu Muli decoction, a Chinese herbal formula, for the treatment of insomnia: a systematic review and meta-analysis. Medicine. 2020;99(40):e22462. doi:10.10 97/MD.0000000000022462

30. Liu Y, Wang W, Chen Y, et al. Simultaneous quantification of nine components in the plasma of depressed rats after oral administration of Chaihu-Shugan-San by ultra-performance liquid chromatography/ quadrupole-time-of-flight mass spectrometry and its application to pharmacokinetic studies. J Pharm Biomed Anal. 2020;186:113310. doi:10.1016/j.jpba.2020.113310

31. Yan Y, Li Q, Du HZ, et al. Determination of five neurotransmitters in the rat brain for the study of the hypnotic effects of Ziziphi Spinosae Semen aqueous extract on insomnia rat model by UPLC-MS/MS. Chin J Nat Med. 2019;17(7):551-560. doi:10.1016/S1875-5364(19) 30077-9

32. He LY, Hu MB, Li RL, et al. The effect of protein-rich extract from Bombyx Batryticatus against glutamate-damaged PC12 cells via regulating $\gamma$-aminobutyric acid signaling pathway. Molecules. 2020;25 (3):553. doi:10.3390/molecules 25030553

33. Zhao HJ, Luo X, Shi YC, et al. The efficacy of fecal microbiota transplantation for children with Tourette syndrome: a preliminary study. Front Psychiatry. 2020;11:554441. doi:10.3389/fpsyt.2020. 554441

34. Zhao H, Shi Y, Luo X, Peng L, Yang Y, Zou L. The effect of fecal microbiota transplantation on a child with Tourette syndrome. Case Rep Med. 2017;2017:6165239. doi:10.1155/2017/6165239

35. Liao JF, Cheng YF, Li SW, et al. Lactobacillus plantarum PS128 ameliorates 2,5-Dimethoxy-4-iodoamphetamine-induced tic-like behaviors via its influences on the microbiota-gut-brain-axis. Brain Res Bull. 2019;153:59-73. doi:10.1016/j.brainresbull.2019.07.027

36. Jiao L, Li Y, Zhang Y, et al. Degradation kinetics of 6"'-p-coumaroylspinosin and identification of its metabolites by rat intestinal flora. J Agric Food Chem. 2017;65(22):4449-4455. doi:10.1021/acs. jafc.7b01486

37. Wu L, Yan Q, Chen F, Cao C, Wang S. Bupleuri radix extract ameliorates impaired lipid metabolism in high-fat diet-induced obese mice via gut microbia-mediated regulation of FGF21 signaling pathway. Biomed Pharmacother. 2021;135:111187. doi:10.1016/j. biopha.2020.111187

38. Bai YF, Wang SW, Wang XX, et al. The flavonoid-rich Quzhou Fructus Aurantii extract modulates gut microbiota and prevents obesity in high-fat diet-fed mice. Nutr Diabetes. 2019;9(1):30. doi:10.1038/s41387-019-0097-6

39. Tan SRS, Eser BE, Han J. Gut metabolism of furanocoumarins: proposed function of Co O-methyltransferase. ACS Omega. 2020;5 (47):30696-30703. doi:10.1021/acsomega.0c04879

40. Casey AB, Canal CE. Classics in chemical neuroscience: aripiprazole. ACS Chem Neurosci. 2017;8(6):1135-1146. doi:10.10 21/acschemneuro.7b00087 


\section{Publish your work in this journal}

Neuropsychiatric Disease and Treatment is an international, peerreviewed journal of clinical therapeutics and pharmacology focusing on concise rapid reporting of clinical or pre-clinical studies on a range of neuropsychiatric and neurological disorders. This journal is indexed on PubMed Central, the 'PsycINFO' database and CAS, and is the official journal of The International Neuropsychiatric Association (INA). The manuscript management system is completely online and includes a very quick and fair peer-review system, which is all easy to use. Visit http://www.dovepress.com/testimonials.php to read real quotes from published authors.

Submit your manuscript here: https://www.dovepress.com/neuropsychiatric-disease-and-treatment-journal 